\title{
POWERS OF IDEALS AND FIBERS OF MORPHISMS
}

\author{
DAVID EISENBUd AND Joe HARRIS
}

\begin{abstract}
Let $X \subset \mathbb{P}^{n}=\mathbb{P}_{F}^{n}$ be a projective scheme over a field $F$, and let $\phi: X \rightarrow Y$ be a finite morphism. Our main result is a formula, in terms of global data, for the maximum of reg $\phi^{-1}(y)$, the Castelnuovo-Mumford regularity of a fiber of $\phi$ over a point $y \in Y$, where the fiber $\phi^{-1}(y)$ is considered as a subscheme of $\mathbb{P}^{n}$.

For example, Cutkosky-Herzog-Trung [1999] and Kodiyalam [2000] showed that for any homogeneous ideal $I$ in a standard graded algebra $S, \operatorname{reg} I^{t}$ can be written as $d t+\epsilon$ for some non-negative integers $d, \epsilon$ and all large $t$. If $I$ contains a power of $S_{+}$and is generated by forms of a single degree, then it determines a morphism $\phi: X:=\operatorname{Proj}(S) \rightarrow Y:=\mathbb{P}^{m}$ as above. In this situation our theorem states that the maximum of the regularities of the fibers of $\phi$ is precisely $\epsilon+1$.

Our formula also gives new information about powers of ideals generated by generic forms in polynomial rings.
\end{abstract}

\section{Introduction}

In this note, all schemes will be projective over an arbitrary field $F$. For any projective scheme $X \subset \mathbb{P}^{n}$ we write $S_{X}$ for the homogeneous coordinate ring of $X$, and $I_{X}$ for the homogeneous ideal of $X$. We denote by reg $X$ the Castelnuovo-Mumford regularity of $I_{X}$ (if $X=\mathbb{P}^{n}$ we make the convention that $\operatorname{reg} X=1$ ).

If $\phi: X \rightarrow Y$ is a finite morphism, then the degree of the fiber $X_{y}=\phi^{-1}(y)$ is a semicontinuous function of $y \in Y$, and is thus bounded. It follows that the CastelnuovoMumford regularity of $X_{y}$, where $X_{y}$ considered as a subscheme of $\mathbb{P}^{n}$, is also bounded. Our main result in the form of Corollary 2.2, gives an algebraic formula for $\max \operatorname{reg}\left(X_{y}\right)$ in terms of global data.

A particularly interesting case occurs when $\phi$ is a morphism induced by a linear projection $\phi: \mathbb{P}^{n} \rightarrow \mathbb{P}^{s}$.

Theorem 0.1. Let $X \subset \mathbb{P}^{n}$ be a projective scheme with homogeneous coordinate ring $S_{X}$, and let $\phi: X \rightarrow \mathbb{P}^{s}$ be a linear projection whose center does not meet $X$, defined by an $s+1$-dimensional vector space of linear forms $V$. Let $I \subset S_{X}$ be the ideal generated by $V$, and let $\mathfrak{m}$ be the maximal homogeneous ideal of $S_{X}$. The maximum of the Castelnuovo-Mumford regularities of the fibers of $\phi$ over closed points of $\mathbb{P}^{s}$ is one more than the least $\epsilon$ such that, for large $t$,

$$
\mathfrak{m}^{t+\epsilon} \subset I^{t} .
$$

In the situation of Theorem 0.1 the number $t+\epsilon$ is equal, for large $t$, to the Castelnuovo-Mumford regularity of $I^{t}$ or the corresponding ideal sheaf (see $\S 1$ ). Thus Theorem 0.1 clarifies the following beautiful result of Cutkosky-Herzog-Trung [1999], Kodiyalam[2000], and Trung-Wang [2005], at least in a special case.

Received by the editors July 21, 2008. 
Theorem 0.2. If $I$ is a homogeneous ideal in the polynomial ring $S=F\left[x_{0}, \ldots, x_{n}\right]$, and $M$ is a finitely generated graded module over $S$, then there are non-negative integers $d, \epsilon$ such that

$$
\operatorname{reg}\left(I^{t} M\right)=d t+\epsilon \quad \text { for all } t \gg 0 .
$$

If $I$ is generated by forms of a single degree $\delta$ and contains a nonzerodivisor on $M$, then $d=\delta$. More generally, Kodiyalam [2000] proves that $d$ is the smallest number $\delta$ such that $I^{t} M=I_{\leq \delta} I^{t-1} M$ for large $t$, where $I_{\leq \delta}$ denotes the ideal generated by the elements of $I$ having degree at most $\delta$. In a paper now in preparation, Eisenbud and Ulrich show, in the special case where $I$ is primary to the maximal homogeneous ideal and is generated by forms of a single degree (the case relevant to this paper), the condition $t \gg 0$ can be made effective: it suffices in this situation to take

$$
t \geq \max \left(\frac{1+\operatorname{reg}(M)}{d}, N\right)
$$

where $N$ is the regularity of the Rees module $\mathcal{R}(I, M)$ with respect to ideal of the Rees ring $\mathcal{R}(I)$ generated by the variables corresponding to generators of $I$.

By contrast, the value of $\epsilon$ has been mysterious. Theorem 0.1 gives an interpretation of $\epsilon$ in a special case. This seems to be new even for ideals generated in a single degree in a polynomial ring in 2 variables, where Corollary 2.2 , applied with $n=1$ and $X=\mathbb{P}^{1}$, yields the following.

Corollary 0.3. Suppose that $I \subset F[x, y]$ is an ideal generated by a vector space $V$ of forms of degree $d$, and that $F$ is algebraically closed. Assume that the greatest common divisor of the forms in $V$ is 1 . For $V^{\prime} \subset V$, let $r_{V^{\prime}}$ be the degree of the greatest common divisor of the forms in $V^{\prime}$, and let

$$
r:=\max \left\{r_{V^{\prime}} \mid V^{\prime} \subset V \text { a subspace of codimension } 1\right\} .
$$

If $t \gg 0$, then $\operatorname{reg} I^{t}=d t+r-1$.

Proof. The integer $\epsilon$ in Corollary 2.2 is the same as that in Theorem 0.2. The space $V$, considered as a linear series $V \subset H^{0}\left(\mathcal{O}_{\mathbb{P}^{1}}(d)\right)$ defines a morphism $\phi$ from $\mathbb{P}^{1}$ to $\mathbb{P}(V)$. A point in the target space, and thus a fiber of the morphism, is defined by codimension 1 subspaces $V^{\prime}$ of $V$. The saturated ideal of the fiber is generated by the greatest common divisor of the elements of $V^{\prime}$, and the regularity of the fiber is thus the degree $r_{V^{\prime}}$ of this greatest common divisor.

The corresponding result holds in the case of polynomial rings in more variables, (and also follows from Theorem 0.1) if we assume that $I$ is primary to the maximal homogeneous ideal and redefine $r_{V^{\prime}}$ to be the maximal degree in which the local cohomology module $H_{\mathfrak{m}}^{1}\left(R /\left(V^{\prime} R\right)\right)$ is nonzero (Proposition 1.2). However, the case of two variables, Corollary 0.3 is special in that, when the morphism $\phi$ defined in its proof is birational, the number $r$ can be interpreted as the maximum multiplicity of a point on the image curve $\phi\left(\mathbb{P}^{1}\right)$.

The first author and Roya Beheshti [2008] have conjectured that the regularity of every fiber of a general linear projection of a smooth projective variety $X$ to $\mathbb{P}^{\operatorname{dim} X+c}$, for $c \geq 1$, is bounded by $1+(\operatorname{dim} X) / c$. Translating this conjecture by means of Theorem 0.1, we get: 
Conjecture 0.1 (Beheshti-Eisenbud [2008]). Let $R$ be a standard graded F-algebra of dimension $n+1$, and let $\mathfrak{m}$ be the maximal homogeneous ideal of $R$. Suppose that $R$ is a domain with isolated singularity. If $F$ is infinite and $I \subset R$ is an ideal generated by $n+1+c$ general linear forms, then

$$
\mathfrak{m}^{t+\epsilon} \subset I^{t} \quad \text { for all } t \gg 0
$$

holds with $\epsilon=\lfloor n / c\rfloor$.

It is easy to see that Conjecture 0.1 holds if $c>n$, and it is known to hold in many other cases as well (see Beheshti-Eisenbud [2008] for a survey), This also gives some new information about ideals generated by generic forms of higher degree. The following is a typical example. Amazingly, we can say no more than this even if we assume that $R$ is the polynomial ring $F\left[x_{0}, \ldots, x_{n}\right]$.

Corollary 0.4. Suppose that $R$ is a standard graded algebra of dimension $n+1$ over a field of characteristic 0 , and that $R$ has at most an isolated singularity. If $I=$ $\left(f_{1}, \ldots, f_{n+2}\right)$ is an ideal generated $n+2$ generic forms of degree $d$, and $n \leq 14$, then

$$
\mathfrak{m}^{t+n} \subset I^{t} \text { for all } t \gg 0
$$

Proof. The linear series given by $f_{1}, \ldots, f_{n+2}$ defines a generic linear projection of Proj $R$. By Mather [1971], generic projections in this range of dimensions are stable maps. Mather [1973] gives a local classification of the multigerms of such stable maps, from which it follows that the degree of the fibers, and thus their regularities, are bounded by $n+1$. The desired formula now follows from Theorem 0.1 .

We do not currently know any function $\epsilon$ of $\operatorname{dim} R$ and $c$ alone that makes the formula in Conjecture 0.1 true. But there is an elementary estimate, whose proof we will give in $\S 1$ :

Proposition 0.5. Let $R$ be a standard graded $F$-algebra of dimension $n+1$, and let $\mathfrak{m}$ be the maximal homogeneous ideal of $R$. If $I \subset R$ is an ideal generated by linear forms, and if $R / I$ has finite length, then

$$
\mathfrak{m}^{t+\epsilon} \subset I^{t} \quad \text { for all } t \gg 0
$$

holds with $\epsilon=\operatorname{reg} R-1$. If $X=\operatorname{Proj} R$ is geometrically reduced and connected in codimension 1, then the same formula holds with $\epsilon=\operatorname{deg} X-\operatorname{codim} X$.

It was conjectured by the first author and Shiro Goto in [1984] that if $X=\operatorname{Proj} R$ is geometrically reduced and connected in codimension 1 , then $\operatorname{reg} X \leq \operatorname{deg} X-\operatorname{codim} X+$ 1 , which would say that the first bound given is always sharper than the second, as well as more general.

In Section 1 we prove a sharp form of Theorem 0.2 in the special case of interest for this paper. We also give the proof of a generalization of Proposition 0.5. Section 2 contains our main result, from which we derive Theorem 0.1.

\section{The Regularity of Powers}

Throughout this paper we write $S=F\left[x_{0}, \ldots, x_{n}\right]$ and set $\mathfrak{m}=\left(x_{0}, \ldots, x_{n}\right)$, the homogeneous maximal ideal.

In the case of most interest for this paper, Theorem 0.2 can be strengthened by an elementary argument, as follows. The result sharpens Theorem 1.1 of Geramita, 
Gimigliano and Pitteloud [1995], which seems to have been the first paper to consider such questions, and Theorem 4 of Chandler [1997]. Part 2 appeared previously, with a less elementary proof, in Corollary 7.5 of Eisenbud-Huneke-Ulrich [2006] (their method gives a similar result in the case of a 1-dimensional ideal with linear presentation.)

Proposition 1.1. Let $M$ be a finitely generated graded $S$-module generated in degree 0 , and let $I \subset S$ be a homogeneous ideal generated by forms of degree d. If $M / I M$ has finite length, but $M$ does not, then we may write

(1) $\operatorname{reg} M / I^{t} M=d t+f_{t}-1$, with $f_{1} \geq f_{2} \geq \cdots \geq 0$.

(2) $\operatorname{reg} I^{t} M=d t+e_{t}$, with $e_{1} \geq e_{2} \geq \cdots \geq 0$.

Moreover, $e:=\inf \left\{e_{t}\right\}=\inf \left\{f_{t}\right\}$, and we have $\operatorname{reg} I^{t} M=d t+e$ for $t \gg 0$.

Proof. We first prove the inequalities of part 1. Since $M / I^{t} M$ has finite length the assertion $\operatorname{reg} M / I^{t} M=d t+f_{t}-1$ means that $f_{t}$ is the smallest number such that

(*) $I^{t} M$ contains all the graded components of $M$ with degree $\geq d t+f_{t}$.

By our hypotheses on the degrees of generators of $M$ and $I$, condition (*) is equivalent to the assertion $\mathfrak{m}^{f_{t}} I^{t} M=\mathfrak{m}^{d t+f_{t}} M$. (A priori we have $\mathfrak{m}^{f_{t}} I^{t} M \subset \mathfrak{m}^{d+f_{t}} I^{t-1} M \subset$ $\mathfrak{m}^{d t+f_{t}} M$, so if $\operatorname{reg} M / I^{t} M \leq d t+f_{t}$ then these three terms are all equal.)

From these equalities we deduce

$$
\mathfrak{m}^{f_{t}} I^{t+1} M=I \mathfrak{m}^{f_{t}} I^{t} M=I \mathfrak{m}^{d+f_{t}} I^{t-1} M=\mathfrak{m}^{d+f_{t}} I^{t} M=\mathfrak{m}^{d(t+1)+f_{t}} M
$$

so $f_{t} \geq f_{t+1}$. Considering the degrees of the generators of $I$ and $M$, and using the fact that $M$ does not have finite length, we see that $f_{t} \geq 0$ for each $t$, completing the proof of part 1.

Turning to the assertion of part 2, it is obvious from the consideration of degrees, and the hypothesis that $M$ does not have finite length, that $e_{t} \geq 0$. To prove that $e_{t} \geq e_{t+1}$, let $N$ be the largest submodule of finite length in $\bar{M}$. If $N=0$, then since $M / I^{t} M$ has finite length, we see from the local cohomology characterization of regularity that

$$
\operatorname{reg} I^{t} M=\max \left(\operatorname{reg} M, 1+\operatorname{reg} M / I^{t} M\right)
$$

so part 2 follows from part 1 in this case. The hypothesis that $M$ does not have finite length implies that $\operatorname{reg} M / I^{t} M$ increases without bounds, so for large $t$ we will have $e_{t}=f_{t}$.

We can reduce the general case to the case $N=0$ by considering the exact sequence

$$
0 \rightarrow I^{t} M \cap N \rightarrow I^{t} M \rightarrow I^{t}(M / N) \rightarrow 0 .
$$

Since $I^{t} M \cap N$ has finite length, while $I^{t}(M / N)$ has no finite length submodule except 0 ,

$$
\operatorname{reg} I^{t} M=\max \left(\operatorname{reg}\left(I^{t} \cap N\right), \operatorname{reg}\left(I^{t}(M / N)\right)\right) .
$$

If we replace $t$ by $t+1$ the term $\operatorname{reg}\left(I^{t} \cap N\right)$ does not increase, while $\operatorname{reg}\left(I^{t}(M / N)\right)$ increases by at most $d$, proving that $e_{t} \geq e_{t+1}$. Because $\operatorname{reg}\left(I^{t}(M / N)\right)$ grows without bound, it eventually dominates, and we again get $e_{t}=f_{t}$ for large $t$.

We remark that Proposition 1.1 need not hold - even for ideals with linear resolutionin the case $M=S$, if when $\operatorname{dim} I \geq 2$ : Terai (credited in Conca [2000]) noted that when char $F \neq 2$, the ideal associated to a triangulation of the projective plane has a linear resolution $(\operatorname{reg} I=3)$, but its square does not $\left(\operatorname{reg} I^{2}=7>2 \times 3\right)$. Sturmfels 
[2000] has given characteristic-free examples of such behavior, and Conca [2006], has given examples with reg $I^{n}=n \operatorname{reg} I$ but reg $I^{n+1}>(n+1) \operatorname{reg} I$ for arbitrary $n$.

We now turn to the proof of Proposition 0.5. The first estimate is a Corollary of the following result:

Proposition 1.2. Let $M$ be a graded module of dimension $n$ over a polynomial ring $S=F\left[x_{0}, \ldots, x_{r}\right]$, and let $I$ be an ideal generated by forms of degree $d$ such that $M / I M$ has finite length. For every $t>0$,

$$
\operatorname{reg} M / I^{t} M \leq t d+\operatorname{reg} M+(n-1)(d-1)-1 .
$$

Moreover, equality holds when the generators of I form a regular sequence on $M$.

Proof. If $I$ is generated by a regular sequence on $M$, then one can obtain a resolution of $M / I^{t} M$ by tensoring a resolution of $M$ with one for $S / I^{t}$ (obtained, for example, as an Eagon-Northcott complex) and from this one computes the regularity at once. (This much does not use the hypothesis that $M / I M$ has finite length.)

When $M / I M$ has finite length, we may begin by replacing $I$ by a smaller ideal, generated by a system of parameters of degree $d$ on $M$-in this case, the regularity of $M / I^{t} M$ is simply the degree of the socle, so it can only increase. It is not hard to give an elementary argument using induction on $t$. Alternately, the result of Caviglia [2007] (see also Sidman [2002]) shows that $\operatorname{reg} M / I^{t} M=\operatorname{reg}\left(M \otimes S / I^{t}\right) \leq \operatorname{reg} M+\operatorname{reg} S / I^{t}=$ $\operatorname{reg} M+(t-1) d+(d-1) \operatorname{dim} M$ where the last equality follows from the argument above and the fact that $I$ is generated by a regular sequence on $S$.

Proof of Proposition 0.5. For the first estimate we set $d=1$ in Proposition 1.2 and use the fact that the regularity of $R / I^{t}$ is the largest number $s$ such that $\mathfrak{m}^{s} \not \subset I^{t}$. For the second estimate we first observe that it suffices to do the case where the number of linear forms is $\operatorname{dim} X$ - that is, a fiber of the projection is just the intersection of $X$ with a plane of complementary dimension. Under the hypotheses given, such a plane section of $X$ is a scheme of degree $\operatorname{deg} X$ and is nondegenerate. The latter condition implies that the regularity of the fiber is bounded above by $\operatorname{deg} X-\operatorname{codim} X+1$. Theorem 2.1 now gives the desired equality.

\section{The Fibers of Finite Morphisms}

We now turn to the result that will allow us to give the maximum regularity of the fibers of a finite morphism in terms of global data (Corollary 2.2). Recall that all schemes are assumed to be projective over a field $F$.

Theorem 2.1. Let $\phi: X \rightarrow \mathbb{P}^{s}$ be a finite morphism from a scheme to a projective space. Set $\mathcal{L}=\phi^{*} \mathcal{O}_{\mathbb{P}^{s}}(1)$ and $V=\phi^{*}\left(H^{0} \mathcal{O}_{\mathbb{P}^{s}}(1)\right) \subset H^{0} \mathcal{L}$. Let $M$ be a coherent sheaf on $X$, and let $W \subset H^{0}(M)$ be a vector space of sections. The following are equivalent:

(1) For every integer $t \gg 0$, the map

$$
\operatorname{Sym}_{t}(V) \otimes W \rightarrow H^{0}\left(\mathcal{L}^{t} \otimes M\right)
$$

is surjective.

(2) For every closed point $p \in \mathbb{P}^{s}$, the restriction map

$$
W \rightarrow H^{0}\left(\phi^{-1}(p) \otimes M\right)
$$

is surjective. 
(3) The map of sheaves

$$
\mu: W \otimes \mathcal{O}_{\mathbb{P} s} \rightarrow \phi_{*} M
$$

is surjective

Proof. $1 \Leftrightarrow 3$ : By Serre's Vanishing Theorem, the surjectivity of $\mu$ is equivalent to the surjectivity, for $t \gg 0$, of the map

$$
W \otimes \operatorname{Sym}_{t}(V)=W \otimes H^{0}\left(\mathcal{O}_{\mathbb{P}(V)}(t)\right) \rightarrow H^{0}\left(\phi_{*}(M)(t)\right) .
$$

But

$$
\phi_{*}(M)(t)=\phi_{*}(M) \otimes_{\mathcal{O}_{\mathbb{P}} s} \mathcal{O}_{\mathbb{P}}(t)=\phi_{*}\left(M \otimes_{\mathcal{O}_{X}} \phi^{*} \mathcal{O}_{\mathbb{P}^{s}}(t)\right)=\phi_{*}\left(M \otimes_{\mathcal{O}_{X}} \mathcal{L}^{t}\right) .
$$

Taking global sections, this gives

$$
H^{0}\left(\phi_{*}(M)(t)\right)=H^{0}\left(\phi_{*}\left(M \otimes_{\mathcal{O}_{X}} \mathcal{L}^{t}\right)\right)=H^{0}\left(M \otimes_{\mathcal{O}_{X}} \mathcal{L}^{t}\right),
$$

proving that assertion 1 is equivalent to assertion 3 .

$2 \Leftrightarrow 3$ : Since $\phi_{*} M$ is coherent, the surjectivity of $\mu$ is equivalent by Nakayama's Lemma to the the surjectivity of all the restriction maps $W=W \otimes \mathcal{O}_{\{p\}} \rightarrow\left(\phi_{*} M\right) \otimes$ $\mathcal{O}_{\{p\}}$, where $p$ runs over the closed points of $\mathbb{P}^{s}$ (or just of the image of $X$ ). Using the finiteness of $\phi$, we can make the identifications

$$
\begin{aligned}
\left(\phi_{*} M\right) \otimes \mathcal{O}_{\{p\}} & =H^{0}\left(\left(\phi_{*} M\right) \otimes \mathcal{O}_{\{p\}}\right) \\
& =H^{0}\left(\phi_{*}\left(M \otimes \phi^{*} \mathcal{O}_{\{p\}}\right)\right) \\
& =H^{0}\left(\left(M \otimes \phi^{*} \mathcal{O}_{\{p\}}\right)\right) \\
& =H^{0}\left(M \otimes \mathcal{O}_{\phi^{-1}(p)}\right)
\end{aligned}
$$

so assertion 2 is also equivalent to the surjectivity of $\mu$.

Corollary 2.2. Suppose that $X \subset \mathbb{P}^{n}$ is a projective scheme, and $\phi: X \rightarrow \mathbb{P}^{s}$ is a finite morphism. Set $\mathcal{L}=\phi^{*} \mathcal{O}_{\mathbb{P}^{s}}(1)$ and $V=\phi^{*}\left(H^{0} \mathcal{O}_{\mathbb{P}^{s}}(1)\right) \subset H^{0} \mathcal{L}$. The maximum regularity, as a subscheme of $\mathbb{P}^{n}$, of a fiber of $\phi$ over a closed point of $\mathbb{P}^{s}$, is one more than the minimum integer $\epsilon$ such that

$$
H^{0}\left(\mathcal{O}_{\mathbb{P}^{n}}(\epsilon)\right) \otimes \operatorname{Sym}_{t}(V) \rightarrow H^{0}\left(\mathcal{L}^{t}(\epsilon)\right)
$$

is surjective for $t \gg 0$.

Proof. The regularity of a fiber $\phi^{-1}(p)$ is the smallest integer $t$ such that $H^{i}\left(\mathcal{I}_{\phi^{-1}(p)}(t-\right.$ $i))=0$ for all $i>0$. For a non-empty fiber $Z=\phi^{-1}(p)$ of dimension 0 , only $i=1$ can be of significance, and the regularity of $Z$ is one more than the minimum $\epsilon$ such that $H^{1}\left(\mathcal{I}_{\phi^{-1}(p)}\right)(\epsilon)=0$. Identifying $\mathcal{O}_{Z}$ with $\mathcal{O}_{Z}(d)$, the long exact sequence in cohomology shows that this is the least $\epsilon$ such that the restriction map

$$
H^{0}\left(\mathcal{O}_{\mathbb{P}^{n}}(\epsilon)\right) \rightarrow H^{0}\left(\mathcal{O}_{Z}(\epsilon)\right) \cong H^{0}\left(\mathcal{O}_{Z}\right)
$$

is surjective. The Corollary thus follows from the equivalence $1 \Leftrightarrow 2$ of Theorem 2.1 if we take $M=\mathcal{O}_{\mathbb{P}^{n}}(\epsilon)$ and $W=H^{0}\left(\mathcal{O}_{\mathbb{P}^{n}}(\epsilon)\right)$.

Proof of Theorem 0.1. In Corollary 2.2 take $\mathcal{L}=\mathcal{O}_{X}(1), M=\mathcal{O}_{X}(e)$, and $W=$ $H^{0}(M)$. The projection $\phi$ is finite since the projection center does not meet $X$. 


\section{Acknowledgements}

We are grateful to Craig Huneke, with whom we first discussed the problem of identifying the number $\epsilon$ in Theorem 0.2. After some experiments using Macaulay2 [M2], he suggested the result in Corollary 0.3, which led us to the results of this paper.

\section{References}

[2008] R. Beheshti and D. Eisenbud, Fibers of Generic Projections, arXiv:0806.1928v2.

[2007] G. Caviglia, Bounds on the Castelnuovo-Mumford regularity of tensor products, Proc. Amer. Math. Soc. 135 (2007) 1949-1957.

[1997] K. A. Chandler, Regularity of the powers of an ideal, Comm. Algebra 25 (1997), 37733776.

[2000] A. Conca, Hilbert function and resolution of the powers of the ideal of the rational normal curve (English summary), Commutative algebra, homological algebra and representation theory (Catania/Genoa/Rome, 1998). J. Pure Appl. Algebra 152 (2000) 65-74.

[2006] A. Conca, Regularity jumps for powers of ideals, In Commutative algebra. Geometric, homological, combinatorial and computational aspects. Ed. Alberto Corso, Philippe Gimenez, Maria Vaz Pinto and Santiago Zarzuela. Lect. Notes Pure Appl. Math., 244, Chapman \& Hall/CRC, Boca Raton, FL, , 21-32, 2006.

[1999] S. Cutkosky, J. Herzog, and N.V. Trung, Assymptotic behavior of the Castelnuovo-Mumford regularity, Compositio Math. 118 (1999) 243-261.

[1997] K.A. Chandler, Regularity of Powers of an Ideal, Comm. Alg. 25 (1997) 3773-3776.

[2005] D. Eisenbud, The Geometry of Syzygies, GTM 229, Springer-Verlag, NY 2005.

[1984] D. Eisenbud and S. Goto, Linear free resolutions and minimal multiplicity, J. Algebra 88 (1984), no. 1, 89-133.

[2006] D. Eisenbud, C. Huneke and B. Ulrich, The regularity of Tor and graded Betti numbers, Amer. J. Math. 128 (2006) 573-605.

[1995] A. V. Geramita, A. Gimigliano and Y. Pitteloud, Graded Betti Numbers of some embedded rational $n$-folds, Math. Ann. 301 (1995) 363-380.

[2000] V. Kodiyalam, Asymptotic behavior of Castelnuovo-Mumford regularity, Proc. Amer. Math. Soc. 128 (2000) 407-411.

[M2] D.R. Grayson and M.E. Stillman, MACAULAY2, a software system for research in algebraic geometry, 1993-, available at http://www.math.uiuc.edu/Macaulay2.

[2004] R. Lazarsfeld, Positivity in Algebraic Geometry I, Ergebnisse der Math. Und ihrer Grenzgebiete 48. Springer-Verlag, Berlin, 2004.

[1971] J. N. Mather, Stability of $C^{\infty}$ mappings. VI: The nice dimensions, Proceedings of Liverpool Singularities-Symposium, I (1969/70) 207-253. Lecture Notes in Math. 192, Springer-Verlag, Berlin, 1971.

[1973] J. N. Mather, Generic projections, Ann. of Math. (2) 98 (1973), 226-245.

[2001] T. Römer, Homological properties of bigraded algebras, Illinois J. Math. 45 (2001) 1361-1376.

[2002] J. Sidman, On the Castelnuovo-Mumford regularity of products of ideal sheaves, Adv. Geom 2 (2002) 219-229.

[2000] B. Sturmfels, Four counterexamples in combinatorial algebraic geometry, J. Algebra 230 (2000) 282-294.

[2005] N.V. Trung and H.-J. Wang, On the asymptotic linearity of Castelnuovo-Mumford regularity, J. Pure and Appl. Alg. 201 (2005) 42-48.

Department of Mathematics, University of California, Berkeley, Berkeley, CA 94720

E-mail address: de@msri.org

URL: http://www.msri.org/ de

Department of Mathematics, Harvard University, Cambridge, MA, 02138

E-mail address: harris@math.harvard.edu 